\title{
Avaliação da aplicabilidade de fórmulas preditivas de peso e estatura em homens adultos ${ }^{1}$
}

\author{
Assessment of equations that estimate \\ weight and height in adult men
}

Fabiane Aparecida Canaan REZENDE ${ }^{2}$

Lina Enriqueta Frandsen Paez Lima ROSADO3

Sylvia do Carmo Castro FRANCESCHINNI ${ }^{3}$

Gilberto Paixão ROSADO3

Rita de Cássia Lanes RIBEIRO3

RE S U M O

\section{Objetivo}

Avaliar a validade de fórmulas preditivas de peso e de altura, bem como a composição corporal em homens adultos.

\section{Métodos}

A amostra constituiu-se de 98 homens saudáveis, com idades entre 20 e 58 anos. Para a análise das equações de estimativa de peso e altura, coletaram-se dados de peso, altura, altura do joelho, envergadura, semi-envergadura, circunferências da panturrilha e do braço e dobra cutânea subescapular. Avaliou-se a composição corporal por meio de bioimpedância elétrica.

\section{Resultados}

O peso estimado diferiu significantemente do peso aferido $(p<0,001)$. Apenas a equação de estimativa de altura validada para homens brancos adultos, mostrou-se adequada para estimar a altura. Tanto a medida de envergadura $(r=0,789 ; d=2,67 ; p<0,001)$ quanto a de semi-envergadura $(r=0,790 ; d=2,51 ; p<0,001)$ resultaram em superestimação da altura aferida. Ao calcular o índice de massa corporal, pelo peso e altura estimados por medidas recumbentes, verificou-se que a maioria dos valores estimados superestimou o baixo peso e subestimou o sobrepeso, exceto quando a altura foi estimada pelas equações propostas para homens adultos.

1 Artigo elaborado a partir da dissertação de F.A.C. REZENDE, intitulada "Comparação de métodos para estimativa de peso, altura e composição corporal de homens adultos". Universidade Federal de Viçosa; 2006.

${ }^{2}$ Universidade Federal do Mato Grosso, Faculdade de Nutrição. Av. Fernando Corrêa da Costa, s/n., Coxipó, 78060-900, Cuiabá, MT, Brasil. Correspondência para/Correspondence to: F.A.C. REZENDE. E-mail: <facrezende@ufmt.br>.

${ }^{3}$ Universidade Federal de Viçosa, Departamento de Nutrição e Saúde. Viçosa, MG, Brasil. 
444 | F.A.C. REZENDE et al.

\section{Conclusão}

A equação de estimativa de altura validada para homens brancos adultos foi adequada para a estimativa da estatura em homens adultos jovens e observou-se que a maioria das equações avaliadas apresentaram diferenças significantes. Ressalta-se a importância da validação das equações aqui avaliadas em outros grupos populacionais, além de cautela ao utilizar peso e altura estimados.

Termos de indexação: Adulto. Antropometria. Composição corporal. Pesos e medidas corporais.

\section{A B S T R A C T}

\section{Objective}

The objective of this study was to evaluate the validity of equations that predict weight, height and body composition in adult men.

\section{Methods}

The sample consisted of 98 healthy men aged from 20 to 58 years. In order to analyze the equations, weight, height, knee height, arm span, half-arm span, calf and arm circumference and subscapular skinfold thickness were collected. Body composition was determined by bioimpedance.

\section{Results}

Estimated weights were significantly different from measured weights $(p<0.001)$. The only equation that estimated height properly was that validated for adult Caucasian men. Both arm span $(r=0.789 ; d=2.67$; $p<0.001)$ and half-arm span $(r=0.790 ; d=2.51 ; p<0.001)$ overestimated height. When weight and height estimates were used to calculate body mass index, underweight was overestimated and overweight was underestimated, except when height was estimated with the equations for adult Caucasian men.

\section{Conclusion}

The equation to estimate height validated for adult Caucasian men estimated the height of adult young men properly; the other validated equations presented significant differences. It is important to validate the equations assessed in this study in other population groups, making sure to use the estimated weights and heights to calculate body mass index.

Indexing terms: Anthropometry. Adult. Body weights and measures. Body composition.

\section{N T R O D U ÇÃ O}

Atualmente, observa-se que as alterações do estado nutricional, principalmente em pacientes hospitalizados, comprometem a imunidade e a capacidade funcional, interferindo de maneira negativa na recuperação dos mesmos ${ }^{1}$. Para a oferta de um suporte nutricional adequado a avaliação do estado nutricional é imprescindível, sendo importante que o profissional tenha acesso a técnicas rápidas e de baixo custo e a métodos precisos que forneçam um diagnóstico confiável.

O Índice de Massa Corporal (IMC) é um indicador amplamente utilizado na avaliação do estado nutricional de indivíduos, e é obtido pelo peso dividido pelo quadrado da altura em metros. Vários estudos têm demonstrado a associação entre IMC e mortalidade e também que, a partir de valores extremos, IMC muito baixo ou muito elevado, a mortalidade é aumentada ${ }^{2-5}$.

A avaliação do IMC é necessária para o diagnóstico nutricional, a escolha de medidas terapêuticas e a monitorização do estado de saúde de indivíduos, principalmente daqueles hospitalizados. Considerando a importância do peso e da altura na avaliação do estado de saúde de um indivíduo, medidas recumbentes são freqüentemente utilizadas na estimativa tanto de peso quanto estatura, quando certas condições impedem a obtenção desses valores. É o caso de pacientes que não deambulam ou que possuem algum tipo de problema relacionado à estrutura óssea, como encurtamento de vértebras, osteoporose e cifose ${ }^{6}$. 
O peso é de grande importância na prática clínica devido à sua utilização na avaliação do gasto energético e à sua associação à morbimortalidade. A porcentagem de perda ponderal e a velocidade desta perda podem indicar a gravidade de uma doença e as chances de mortalidade de um indivíduo ${ }^{7-10}$.

As fórmulas preditivas de peso e de altura, normalmente utilizadas na prática clínica, são baseadas em medidas recumbentes, tais como circunferência do braço e da panturrilha, dobra cutânea subescapular, altura do joelho, envergadura e semi-envergadura ${ }^{6,11,12}$. Contudo, muitos cuidados devem ser tomados na obtenção e na interpretação dessas medidas, já que fatores como obesidade, edema e desidratação podem comprometer a sua validade.

Ao considerar que pode ser impossível obter o peso e altura em indivíduos adultos, acometidos por doenças, e que são valores essenciais na avaliação do estado nutricional, este estudo avaliou a validade de fórmulas preditivas de peso e de altura e a composição corporal em homens adultos.

\section{MÉ T O D O S}

Trata-se de um estudo epidemiológico, de caráter transversal, realizado na Divisão de Saúde da Universidade Federal de Viçosa (UFV), Viçosa (MG), durante o período de fevereiro a outubro de 2005.

A amostra foi obtida por livre demanda e constituída de 98 homens, que foram selecionados segundo critérios de inclusão (sexo masculino; idade entre 20 e 60 anos e IMC $<30 \mathrm{~kg} / \mathrm{m}^{2}$ ) e de exclusão (doenças crônicas auto-relatadas, como hipertensão e diabetes; anasarca e edema periférico; indivíduos em diálise; uso de medicamentos que pudessem afetar a composição corporal, como diuréticos, corticosteróides, $\beta$-bloqueadores; e amputação ou paralisia de membros).

O projeto foi aprovado pelo Comitê de Ética em Pesquisa da UFV e todos os indivíduos foram esclarecidos sobre os procedimentos da coleta antes de assinarem o Termo de Consentimento, protocolo n 40503352932.

Todas as avaliações foram realizadas pela manhã. Foram recomendados aos voluntários: jejum alimentar de 10 horas, jejum de bebida alcoólica de 24 horas e a não prática de atividade física por 24 horas.

A avaliação antropométrica incluiu peso, altura, altura do joelho, envergadura e semi-envergadura, circunferências da panturrilha, do braço e da cintura e dobra cutânea subescapular. O peso foi aferido em balança digital com capacidade máxima de $150 \mathrm{~kg}$ e divisão de $100 \mathrm{~g}$, segundo as normas preconizadas por Jellife ${ }^{13}$, com os indivíduos descalços e usando apenas um short. A altura foi aferida com estadiômetro fixo a uma parede sem rodapé, com extensão de $2,00 \mathrm{~m}$, dividido em centímetros e subdividido em milímetros, com visor de plástico e esquadro acoplado a uma das extremidades, segundo as normas preconizadas por Jelliffe ${ }^{13}$. O IMC foi calculado tanto com o peso e altura reais, quanto com as medidas estimadas, pela fórmula: IMC=Peso (kg)/Altura $(m)^{2}$ e a classificação do estado nutricional foi feita segundo os pontos de corte preconizados pela Organização Mundial de Saúde $(\mathrm{WHO})^{14}$. A circunferência da cintura foi aferida com fita flexível e inelástica ao redor da cintura normal ou na menor curvatura localizada entre as costelas e a crista ilíaca, mantendo-a justa, sem comprimir os tecidos. Considerou-se obesidade abdominal quando a circunferência da cintura foi maior ou igual a $94 \mathrm{~cm}^{14}$.

A altura do joelho foi medida na perna esquerda, formando um ângulo de $90^{\circ} \mathrm{com}$ o joelho e o tornozelo, e com o indivíduo na posição supina ${ }^{6}$. Utilizou-se um paquímetro, contendo uma régua fixa com sensibilidade de $0,1 \mathrm{~cm}$, constituído por uma parte fixa, que foi posicionada na superfície plantar do pé (calcanhar) e uma parte móvel, que foi pressionada sobre a cabeça da patela (rótula). A altura do joelho (AJ) foi calculada a partir das seguintes fórmulas para estimar a estatura: 
446 | F.A.C. REZENDE et al.

$$
\begin{aligned}
& \text { Chumlea et al. }{ }^{6} \text { - Idosos } \\
& \text { Altura } 1\left(A_{1}\right)=(2,02 \times A J)-(0,04 \times \text { idade }) \\
& +64,19
\end{aligned}
$$

Chumlea et al. ${ }^{11}$ - Adultos

Etnia negra: Altura $2\left(A_{2}\right)=73,42+(1,79$ $x$ altura do joelho)

Etnia branca: Altura $3\left(A_{3}\right)=71,85+(1,88$ $x$ altura do joelho)

A envergadura ou o comprimento dos braços compreendeu a distância entre as falanges distais dos dedos médios e foi obtida com os braços formando um ângulo de $90^{\circ}$ em relação ao tronco (Altura 4: $A_{4}$ ). A semi-envergadura correspondeu à distância entre o esterno e a falange distal do dedo médio esquerdo (Altura 5: $A_{5}$ ), passando uma fita métrica flexível e inelástica paralelamente à clavícula. A medida total da envergadura e o dobro da medida da semi-envergadura corresponderam à estimativa da altura ${ }^{12}$.

As circunferências foram obtidas segundo as técnicas propostas por Callaway et al. ${ }^{15}$. A circunferência do braço foi medida no braço não dominante, no ponto médio entre o acrômio e o olécrano, e a da panturrilha no perímetro máximo do músculo da panturrilha da perna esquerda, formando um ângulo de $90^{\circ}$ com o joelho e o tornozelo, com o indivíduo sentado e a perna relaxada.

A dobra cutânea subescapular foi obtida pela média de três medidas realizadas por um mesmo avaliador, com um compasso da marca Lange, no lado direito obliquamente ao eixo longitudinal do corpo, logo abaixo do ângulo inferior da escápula ${ }^{16}$

Para estimar o peso, foram medidas a Circunferência da Panturrilha (CPA), a Circunferência do Braço (CB), a Altura do Joelho (AJ) e a Dobra Cutânea Subescapular (DSE), utilizando a seguinte fórmula ${ }^{6}$ :

Peso $\left(P_{E}\right)=(0,98 \times C P A)+(1,16 \times A J)+$ $(1,73 \times C B)+(0,37 \times$ DSE $)-81,69$

A composição corporal foi avaliada pelo método de bioimpedância elétrica tetrapolar, com o aparelho Biodynamics ${ }^{\circledR}$ modelo 310. Apesar de o fabricante recomendar 4 horas de jejum, foram solicitadas 10 horas, segundo recomendações de Slinde \& Rossander-Hulthén ${ }^{17}$. Os indivíduos foram previamente orientados a não realizar exercícios físicos e a não consumir bebidas alcoólicas nas 24 horas anteriores ao teste e urinar 30 minutos antes.

A análise de bioimpedância elétrica tetrapolar (BIA) foi realizada com o indivíduo deitado sobre uma superfície não condutora, na posição supina, com braços e pernas abduzidos a $45^{\circ}$, a partir do corpo. Imediatamente antes da colocação dos eletrodos, as áreas de contato foram limpas com álcool. Um eletrodo emissor foi colocado próximo à articulação metacarpo-falange da superfície dorsal da mão direita e o outro distal do arco transverso da superfície superior do pé direito. Um eletrodo detector foi colocado entre as proeminências distais do rádio e da ulna do punho direito e o outro, entre os maléolos medial e lateral do tornozelo direito, de acordo com as instruções do manual do fabricante.

Os dados obtidos foram analisados no programa SPSS for Windons versão 10.0 (SPSS Inc., Chicago, IL, USA). Todas as variáveis foram testadas quanto à sua normalidade pelo teste de Kolmogorov-Smirnov. Com as variáveis que apresentaram distribuição normal, utilizaram-se o coeficiente de correlação de Pearson e o teste $t$ pareado. Quando as variáveis não apresentaram distribuição normal, foram utilizados o coeficiente de correlação de Spearman e o teste de Wilcoxon. Adotou-se como nível de significância estatística o valor de $p$ menor que 0,05 .

\section{RESULTADOS}

A amostra do estudo constituiu-se de 98 homens adultos e a idade média foi de 33,33 anos, indicando que ocorreu predomínio de adultos jovens. Observou-se ainda a presença de indivíduos com sobrepeso, já que o IMC variou entre 17,32 e $29,67 \mathrm{~kg} / \mathrm{m}^{2}$ e com obesidade abdominal, pois a circunferência da cintura variou entre 65,2 e $106,8 \mathrm{~cm}$ (Tabela 1). 
Com relação às medidas antropométricas, verificou-se que a altura do joelho apresentou alta correlação com a altura; as circunferências da panturrilha e do braço apresentaram boa correlação com o peso aferido $\left(P_{A}\right)$, e a dobra cutânea subescapular se correlacionou mais com a massa de gordura determinada por bioimpedância elétrica (BIA) e IMC (Tabela 2).

As correlações entre o peso e a altura aferidos e estimados foram boas e significantes, sendo $\mathrm{A}_{2}$ e $\mathrm{A}_{3}$ baseadas na altura estimada pelas equações propostas para adultos negros e brancos as mais fortes. Observou-se também que o peso estimado $\left(P_{E}\right)$ diferiu significantemente do peso aferido $\left(P_{A}\right)$. Os valores de altura estimados também diferiram significantemente da altura aferida, com exceção da altura estimada pela equação de Chumlea et al. ${ }^{11}$, proposta para adultos da etnia branca $\left(A_{3}\right)$, que apresentou diferença média próxima de zero. Tanto a medida de envergadura quanto a semi-envergadura resultaram em superestimação da altura aferida, enquanto as outras duas equações $\left(A_{1}\right.$ e $\left.A_{2}\right)$, que utilizam a medida da altura do joelho, resultaram em subestimação (Tabela 3).

Com relação à utilização de peso e/ou altura estimados na obtenção do IMC, observou-se que a maioria das estimativas diferiram significantemente do IMC real, com exceção do IMC 8 e do IMC 10, obtidos a partir do peso aferido e do peso estimado, respectivamente, e da altura 3 $\left(\mathrm{A}_{3}\right)$ estimada pela equação de Chumlea et al. ${ }^{11}$ para homens adultos brancos (Tabela 4).

Tabela 1. Caracterização de homens adultos, segundo a idade e parâmetros antropométricos. Viçosa (MG), 2005.

\begin{tabular}{|c|c|c|c|c|}
\hline Variáveis & Média & DP & Mediana & Mínimo - Máximo \\
\hline Idade $(\text { anos) })^{\#}$ & 33,33 & 10,76 & 29,50 & $20,00-58,00$ \\
\hline Peso aferido (kg) & 73,83 & 9,67 & 73,45 & $54,40-96,40$ \\
\hline Estatura aferida $(\mathrm{cm})$ & 174,94 & 5,58 & 175,00 & $161,80-188,20$ \\
\hline$C C(\mathrm{~cm})$ & 84,76 & 9,54 & 84,50 & $65,20-106,80$ \\
\hline IMC real $\left(\mathrm{kg} / \mathrm{m}^{2}\right)$ & 24,10 & 2,81 & 23,99 & $17,32-29,67$ \\
\hline $\mathrm{AJ}(\mathrm{cm})$ & 54,70 & 2,32 & 54,20 & $48,40-60,60$ \\
\hline Enverg (cm) & 177,62 & 6,89 & 177,05 & $161,80-194,40$ \\
\hline SEM (cm) & 88,73 & 3,57 & 88,50 & $80,50-97,50$ \\
\hline $\mathrm{CB}(\mathrm{cm})$ & 30,98 & 2,74 & 31,20 & $23,60-37,20$ \\
\hline $\mathrm{CPA}(\mathrm{cm})^{\#}$ & 37,90 & 3,83 & 38,00 & $32,00-65,40$ \\
\hline DSE $(\mathrm{mm})$ & 18,29 & 6,16 & 17,58 & $5,00-33,66$ \\
\hline
\end{tabular}

DP: desvio-padrão; CC: circunferência da cintura; AJ: altura do joelho; Enverg: envergadura; SEM: semi-envergadura; CB: circunferência do braço; CPA: circunferência da panturrilha; DSE: dobra cutânea subescapular.

\#Não apresentou distribuição normal.

Tabela 2. Correlação entre medidas utilizadas por Chumlea et al. ${ }^{6,11}$ nas equações preditivas de peso e altura e indicadores do estado nutricional em homens adultos. Viçosa (MG), 2005.

\begin{tabular}{|c|c|c|c|c|}
\hline Variáveis & $\mathrm{CPA}^{\#}$ & $C B$ & DSE & AJ \\
\hline Peso & $0,767^{a *}$ & $0,819^{b^{*}}$ & $0,468^{\mathbf{b}^{\star}}$ & $0,598^{\mathbf{b}^{\star}}$ \\
\hline Altura & $0,336^{2 *}$ & $0,219^{b^{* *} b}$ & $-0,170^{\mathbf{b} * * *}$ & $0,828^{b^{*}}$ \\
\hline IMC & $0,678^{a *}$ & $0,799^{b^{*}}$ & $0,623^{b^{*}}$ & $0,212^{\mathbf{b}^{* *}}$ \\
\hline MMBIA & $0,752^{2 *}$ & $0,749^{b^{*}}$ & $0,187^{b^{* * *}}$ & $0,661^{b^{*}}$ \\
\hline$\%$ GCBIA ${ }^{\#}$ & $0,279^{a *}$ & $0,428^{a^{*}}$ & $0,636^{a^{*}}$ & $0,099^{a^{\star \star \star \star}}$ \\
\hline
\end{tabular}

CPA: circunferência da panturrilha; CB: circunferência do braço; DSE: dobra cutânea subescapular; AJ: altura do joelho; IMC: índice de massa corporal; MMBIA: massa magra (kg) obtida por BIA; \%GCBIA: percentual de gordura corporal obtido por BIA. "Não apresentou distribuição normal.

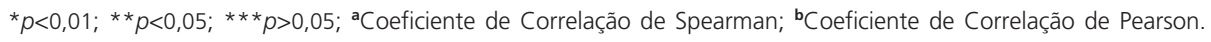


Tabela 3. Comparação entre as médias e correlação de peso e de altura reais com peso e altura estimados em homens adultos. Viçosa (MG), 2005

\begin{tabular}{|c|c|c|c|c|c|c|c|}
\hline \multicolumn{2}{|c|}{ Variáveis } & M & DP & $\mathrm{Md}$ & Diferença média & IC (95\%) & r \\
\hline \multirow{2}{*}{ Peso (kg) } & Aferido & 73,83 & 9,67 & 73,45 & - & - & - \\
\hline & Estimado & 79,29 & 9,58 & $72,18^{a^{\star}}$ & - & - & $0,849^{c^{*}}$ \\
\hline \multirow{6}{*}{ Altura $(\mathrm{cm})$} & Aferida & 174,94 & 5,58 & 175,00 & - & - & - \\
\hline & Altura 1 & 173,35 & $4,72^{b}$ & 172,10 & $-1,59^{*}$ & $-2,21--0,97$ & $0,790^{d^{*}}$ \\
\hline & Altura 2 & 171,33 & $4,16^{\mathbf{b}}$ & 170,43 & $-3,61^{\star *}$ & $-2,97--4,24$ & $0,828^{c^{*}}$ \\
\hline & Altura 3 & 174,68 & $4,37^{\mathbf{b}}$ & 173,74 & $-0,26$ & $-0,88--0,37$ & $0,828^{c^{*}}$ \\
\hline & Altura 4 & 177,62 & $6,89^{b}$ & 177,05 & $+2,67^{*}$ & $1,82-3,52$ & $0,789^{c^{*}}$ \\
\hline & Altura 5 & 177,46 & $7,14^{\mathbf{b}}$ & 177,00 & $+2,51^{*}$ & $1,64-3,38$ & $0,826^{\mathbf{d}^{*}}$ \\
\hline
\end{tabular}

M: média; DP: desvio-padrão; Md: mediana; IC: intervalo de confiança; r: coeficiente de correlação; Altura $1\left(A_{1}\right)=(2,02 \times A J)-(0,04 \times$ idade $)$ $+64,19$; Altura $2\left(A_{2}\right)=73,42+\left(1,79 X\right.$ altura do joelho); Altura $3\left(A_{3}\right)=71,85+\left(1,88 X\right.$ altura do joelho); Altura $4\left(A_{4}\right)=$ envergadura; Altura

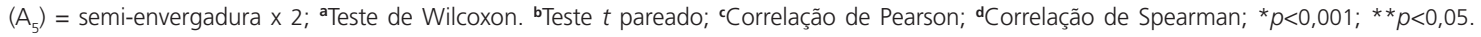

Tabela 4. Teste de comparação de médias entre valores de índice de massa corporal real e índice de massa corporal estimado por medidas recumbentes em homens adultos. Viçosa (MG), 2005.

\begin{tabular}{|c|c|c|c|c|c|}
\hline Variáveis & M & $\mathrm{DP}\left(\mathrm{kg} / \mathrm{m}^{2}\right)$ & Diferença média & IC (95\%) & $r$ \\
\hline IMC Real & 24,10 & 2,81 & - & - & - \\
\hline IMC 1 & 20,76 & 2,43 & $-3,34^{*}$ & $-3,53--3,14$ & $0,938^{a^{*}}$ \\
\hline IMC 2 & 21,26 & 2,49 & $-2,84^{\star}$ & $-3,04--2,62$ & $0,928^{a^{*}}$ \\
\hline IMC 3 & 20,78 & 2,44 & $-3,31^{*}$ & $-3,51--3,12$ & $0,941^{a^{*}}$ \\
\hline IMC 4 & 20,41 & 2,18 & $-3,68^{*}$ & $-4,07--3,29$ & $0,721^{a^{*}}$ \\
\hline IMC 5 & 20,90 & 2,17 & $-3,19^{*}$ & $-3,58--2,80$ & $0,728^{b^{*}}$ \\
\hline IMC 6 & 20,43 & 2,20 & $-3,66^{*}$ & $-4,06--3,27$ & $0,706^{\mathrm{a}^{*}}$ \\
\hline IMC 7 & 25,10 & 2,72 & $0,99^{\star}$ & $0,81-1,17$ & $0,950^{\mathrm{a}^{*}}$ \\
\hline IMC 8 & 24,14 & 2,61 & $0,04^{\star *}$ & $0,13-0,21$ & $0,950^{\mathrm{a}^{*}}$ \\
\hline IMC 9 & 24,67 & 2,33 & $0,57^{\star}$ & $0,19-0,95$ & $0,745^{a^{*}}$ \\
\hline IMC 10 & 23,73 & 2,23 & $-0,09^{\star \star}$ & $-0,09-0,19$ & $0,745^{a^{*}}$ \\
\hline
\end{tabular}

M: média; DP: desvio-padrão; IC: intervalo de confiança; $r$ : coeficiente de correlação. IMC $1=P_{A} /\left(A_{4}\right)^{2}, I M C 2=P_{A} /\left(A_{1}\right)^{2}, I M C 3=P_{A} /\left(A_{5}\right)^{2}$; $I M C 4=P_{E} /\left(A_{4}\right)^{2}, I M C 5=P_{E} /\left(A_{1}\right)^{2}, I M C 6=P_{E} /\left(A_{5}\right)^{2}, I M C 7=P_{A} /\left(A_{2}\right)^{2}, I M C 8=P_{A} /\left(A_{3}\right)^{2}, I M C 9=P_{E} /\left(A_{2}\right)^{2}, I M C 10=P_{E} /\left(A_{3}\right)^{2}, A$ Itura 1 $\left(A_{1}\right)=(2,02 \times A J)-(0,04 \times$ idade $)+64,19$; Altura $2\left(A_{2}\right)=73,42+(1,79 \times$ altura do joelho $)$; Altura $3\left(A_{3}\right)=71,85+(1,88 \times$ altura do joelho $)$; Altura $4\left(A_{4}\right)=$ envergadura; Altura $\left(A_{5}\right)=$ semi-envergadura $\times 2$.

${ }^{1}$ Teste $t$ pareado; a Correlação de Pearson; ${ }^{b}$ Correlação de Spearman; ${ }^{*} p<0,001 ;{ }^{* *} p>0,05$.

Realizou-se a classificação do estado nutricional pelos valores de IMC real e estimado, e verificou-se que os valores de IMC baseados na altura estimada, tanto pela medida de envergadura e de semi-envergadura quanto pela equação proposta para idosos por Chumlea et al. ${ }^{6}$, superestimaram o número de indivíduos com baixo peso e eutróficos e subestimaram o sobrepeso (Tabela 5).

Os índices de massa corporal, baseados na altura estimada pelas equações propostas para homens adultos (IMC 7, 8, 9 e 10) demonstraram- -se mais adequados comparados aos outros, sendo que o IMC 8, baseado no peso aferido e na altura estimada pela equação para adultos brancos $\left(A_{3}\right)$ apresentou melhor concordância com as porcentagens de indivíduos encontradas nas categorias do IMC real (Tabela 5).

\section{DIS CUSS Ã O}

Na literatura internacional, o número de estudos com adultos é relativamente pequeno. A maioria dos estudos com medidas recumbentes 
Tabela 5. Porcentagem de homens adultos com baixo peso, eutrofia e sobrepeso, segundo a classificação pelo IMC real e pelos valores de IMC estimados por meio de medidas recumbentes. Viçosa (MG), 2005

\begin{tabular}{|c|c|c|c|}
\hline \multirow{2}{*}{ Variáveis } & \multicolumn{3}{|c|}{ IMC $\left(\mathrm{kg} / \mathrm{m}^{2}\right)$ Estado nutricional (WHO, 1998) } \\
\hline & Baixo Peso $<18,0$ & Eutrofia $18,5-24,99$ & Sobrepeso 25,0 - 29,99 \\
\hline IMC Real (\%) & 2,0 & 61,2 & 36,7 \\
\hline IMC 1 (\%) & 18,4 & 77,6 & 4,1 \\
\hline IMC 2 (\%) & 12,2 & 79,6 & 8,2 \\
\hline IMC $3(\%)$ & 18,4 & 77,6 & 4,1 \\
\hline IMC 4 (\%) & 19,4 & 79,6 & 1,0 \\
\hline IMC 5 (\%) & 14,3 & 82,7 & 3,1 \\
\hline IMC 6 (\%) & 19,4 & 79,6 & 1,0 \\
\hline IMC 7 (\%) & 0,0 & 51,0 & 49,0 \\
\hline IMC 8 (\%) & 2,0 & 57,2 & 40,8 \\
\hline IMC 9 (\%) & 0,0 & 54,1 & 45,9 \\
\hline IMC $10(\%)$ & 2,0 & 73,5 & 24,5 \\
\hline
\end{tabular}

IMC: índice de massa corporal; IMC $1=P A /\left(A_{4}\right)^{2}, I M C 2=P_{A} /\left(A_{1}\right)^{2}, I M C 3=P_{A} /\left(A_{5}\right)^{2}, I M C 4=P_{E} /\left(A_{4}\right)^{2}, I M C 5=P_{E} /\left(A_{1}\right)^{2}, I M C 6=P_{E} /\left(A_{5}\right)^{2}, I M C$ $7=P_{A} /\left(A_{2}\right)^{2}, I M C 8=P_{A} /\left(A_{3}\right)^{2}, I M C 9=P_{E} /\left(A_{2}\right)^{2}, I M C 10=P_{E} /\left(A_{3}\right)^{2} ;$ Altura $1\left(A_{1}\right)=(2,02 \times A J)-(0,04 \times$ idade $)+64,19 ;$ Altura $2\left(A_{2}\right)=73,42+(1,79$ $X$ altura do joelho); Altura $3\left(A_{3}\right)=71,85+\left(1,88 \times\right.$ altura do joelho); Altura $4\left(A_{4}\right)=$ envergadura; Altura $\left(A_{5}\right)=$ semi-envergadura $\times 2$.

envolve as populações infantil e idosa, e a medida antropométrica mais estudada tem sido a altura. Han \& Lean ${ }^{18}$ verificaram que $95 \%$ dos erros da altura estimada pela altura do joelho foram de até $6,5 \mathrm{~cm}$ e que a envergadura resultou em erros inaceitáveis em amostra de 78 homens e 82 muIheres, entre 17 e 70 anos, assim como no presente estudo em que a estimativa da estatura pelas medidas de envergadura e de semi-envergadura resultou em superestimação.

No Brasil, apenas Sampaio et al. ${ }^{19}$ investigaram a validade das equações aqui utilizadas em 75 homens adultos, constatando que as estimativas da altura fornecidas pela equação de Chumlea et al. ${ }^{6}$, proposta para idosos, diferiram significantemente da medida aferida. No presente estudo, observou-se que a utilização da equação de Chumlea et al. ${ }^{6}$ resultou também em superestimação da estatura. Ainda no estudo de Sampaio et al. ${ }^{19}$, verificou-se que a diferença média entre o peso estimado e peso aferido $(-1,16 \mathrm{~kg})$ não foi significante $(p=0,25)$, ao contrário deste estudo em que a diferença foi significante $(p<0,001)$.

Assim como neste estudo, a superestimação do baixo peso também foi verificada por Sampaio et al. ${ }^{19}$ ao utilizarem a altura estimada pela equação de Chumlea et al. ${ }^{6}$ no cálculo do
IMC, demonstrando novamente a inadequação da referida equação.

No estudo de De Lucia et al. ${ }^{20}$, realizado com quatro grupos étnicos da Etiópia, a relação entre envergadura e altura foi influenciada pelo sexo e pela etnia, e a utilização da envergadura para cálculo do IMC resultou em superestimação da desnutrição em 1706 indivíduos de ambos os sexos, com idades entre 18 e 50 anos, resultado este também verificado no presente estudo.

Diante dos resultados, observa-se que a etnia e a idade são fatores determinantes na validade das equações preditivas de peso e de estatura. Quando se utilizou a equação de Chumlea et al. ${ }^{11}$, validada para homens adultos e brancos, observou-se que a estimativa de estatura foi mais precisa, ao contrário das estimativas baseadas em equações previamente validadas em negros e em idosos que resultaram em diferenças significantes.

Como a avaliação do estado nutricional é de extrema importância para a determinação das condutas clínico-nutricionais, deve-se ter muita cautela ao utilizar estimativas como as apresentadas neste estudo. Se estimativas que resultam em superestimação do baixo peso ou desnutrição são utilizadas, há maior risco de adoção de uma conduta equivocada, interferindo diretamente na 
saúde do indivíduo, principalmente, daqueles hospitalizados.

O diagnóstico de desnutrição pode indicar aumento das necessidades dietéticas, resultando em maior oferta de energia e nutrientes por meio da alimentação. Entretanto, caso haja sobrecarga de energia, quadros como hiperglicemia, esteatose hepática, arritmias cardíacas, edema, falência respiratória e hemólise podem ser desencadeados 21,22 .

\section{CONCLUSÃ O}

A maioria das equações utilizadas neste estudo não foi adequada para estimar peso e altura de homens adultos, já que os valores de peso e de altura estimados diferiram significantemente dos valores aferidos. Considerando todas as equações aqui avaliadas, observou-se que apenas a equação proposta para homens adultos brancos mostrou-se adequada para estimar a altura dos indivíduos avaliados na amostra.

Devido à escassez de estudos com relação à estimativa de peso por meio da equação aqui avaliada, ainda não é possível estabelecer uma discussão mais profunda acerca de sua aplicabilidade, sendo necessários mais estudos que venham a esclarecer sobre o assunto e verificar se essas diferenças persistem em outros grupos populacionais.

Conhecer a acurácia dessas equações é fundamental para verificar a sua aplicabilidade na prática clínica, e estudos de validação poderão, futuramente, contribuir para o diagnóstico correto e o direcionamento adequado das intervenções em indivíduos a partir de estimativas mais acuradas do peso e da altura daqueles nos quais a coleta dessas medidas é impossibilitada.

\section{COLABORADORES}

F.A.C. REZENDE contribuiu com a concepção do trabalho, a coleta dos dados, análise dos dados e a redação do artigo. L.E.F.P.L. ROSADO e G.P. ROSADO contribuíram com a concepção do trabalho e a redação do artigo. S.C.C. FRANCESCHINNI contribuiu com os aspectos metodológicos e os testes estatísticos. R.C.L. RIBEIRO contribuiu com as análises estatísticas.

\section{REFERÊ NCIAS}

1. Waitzberg DL, Caiaffa WT, Correia MI. Hospital malnutrition: the Brazilian national survey (IBRANUTRI): a study of 4000 patients. Nutrition. 2001; 17(7-8):573-80

2. Calle EE, Thun MJ, Petrelli JM, Rodriguez C, Heath CW. Body-mass index and mortality in a prospective cohort of U.S. adults. N Engl J Med. 1999; 341(5):1097-105

3. Manson JE, Willett WC, Stampfer MJ, Colditz GA, Hunter DJ, Hankinson SE, et al. Body weight and mortality among women. N Engl J Med. 1995; 333(11):677-85.

4. Stevens J, Cai J, Pamuk ER, Williamson DF, Thun MJ, Wood JL. The effect of age on the association between body-mass index and mortality. N Eng J Med. 1998: 338(1):1-7.

5. Wienpahl J, Ragland DR, Sidney S. Body mass index and 15-year mortality in a cohort of black men and women. J Clin Epidemiol. 1990; 43(9): 949-60.

6. Chumlea WA, Roche AF, Mukherjee D. Nutritional assessment of the elderly through anthropometry. Columbus (OH): Ross Laboratories; 1987.

7. Baxter JP. Problems of nutritional assessment in acute setting. Proc Nutr Soc. 1999; 58(1):39-46.

8. Gregg EW, Gerzoff RB, Thompson TJ, Williamson DF. Intentional weight loss and death in overweight and obese U.S. adults 35 years of age and older. Ann Intern Med. 2003; 138(5):383-9.

9. Lankisch PG, Gerzmann MJ, Gerzmann F, Lehnick D. Unintentional weight loss: diagnosis and prognosis. The first prospective follow-up study from a secondary referral centre. J Intern Med. 2001; 249(1):41-6

10. Prescott E, Almdal T, Mikkelsen $\mathrm{KL}$, Tofteng $\mathrm{CL}$, Vestbo J, Lange P. Prognostic value of weight change in chronic obstructive pulmonary disease: results from the Copenhagen City Heart Study. Eur Respir J. 2002; 20(3):539-44.

11. Chumlea WC, Guo SS, Steinbaugh ML. Prediction of stature from knee height for black and White adults and children with application to mobility impaired or handicapped persons. J Am Diet Assoc. 1994; 94(12):1385-88. 
12. Kwok T, Writelow MN. The use of armspan in nutritional assessment of the elderly. J Am Geriatr Soc. 1991; 9(5):455-547.

13. Jelliffe DB. The assessment of the nutritional status of the community. Geneva: WHO; 1966.

14. World Health Organization. Obesity: preventing and managing the global epidemic. Report of a WHO Consulation. Geneva: WHO; 1998. Technical Report Series, 894.

15. Callway CW, Chumlea WC, Bouchard C, Himes JH, Lohman TG, Martin AD, et al. Circumferences. In: Lohman TG, Roche AF, Martorell R. Anthropometric standardization reference manual. Champaign (IL): Human Kinetics; 1988. p.39-54.

16. Durnin JVGA, Womersley J. Body fat assessed from total body density and its estimation from skinfold thickness: measurements on 481 men and women aged from 16 to 72 years. Br J Nutr. 1974; 32(1): 77-97.

17. Slinde F, Rossander-Huthén L. Bioelectrical impedance: effect of 3 identical meals on diurnal impedance variation and calculation of body composition. Am J Clin Nutr. 2001; 74(4):474-8.
18. Han TS, Lean ME. Lower leg length as an index of stature in adults [abstract]. Int J Obes Relat Metab Disord. 1996; 20(1):21-7.

19. Sampaio HAC, Melo MLP, Almeida PC, Benevides ABP. Aplicabilidade das fórmulas de estimativa de peso e estatura para idosos e adultos. Rev Bras Nutr Clin. 2002; 17(4):117-21.

20. De Lucia E, Lemma F, Tesfaye F, Demisse T, Ismail S. The use of armspan measurement to assess the nutritional status of adults in four Ethiopian ethnic groups [abstract]. Eur J Clin Nutr. 2002; 56(2): 91-5.

21. Parrish CR, MCcray SF. Nutrition support for the mechanically ventilated patient. Crit Care Nurse. 2003; 23(1):77-80.

22. Roubenoff R. Inflammatory and hormonal mediators of cachexia. J Nutr. 1997; 127(5 Suppl): S1014-16.

Recebido em: 20/6/2007

Versão final reapresentada em: 24/4/2009

Aprovado em: 5/5/2009 